\title{
Quasi-geodesic Segments and Gromov Hyperbolic Spaces
}

\author{
MARIO BONK ${ }^{\star}$ \\ Institut für Analysis, Technische Universität Braunschweig, Pockelsstraße 14, D-38106 \\ Braunschweig, Germany; and \\ Department of Mathematics, University of Michigan, Ann Arbor, MI 48109, U.S.A. \\ e-mail:M.Bonk@ws.rz.tu-bs.de
}

(Received: 20 February 1995)

\begin{abstract}
It is known that for a geodesic metric space hyperbolicity in the sense of Gromov implies geodesic stability. In this paper it is shown that the converse is also true. So Gromov hyperbolicity and geodesic stability are equivalent for geodesic metric spaces.
\end{abstract}

Mathematics Subject Classification (1991): 53C23.

Key words: Hyperbolicity, stability, quasi-geodesics.

\section{Introduction}

Hyperbolic spaces are geodesically stable in the following sense. Each quasigeodesic segment (being defined as the bilipshitz image of an interval) is contained in a neighborhood of a geodesic segment (an isometric image of an interval) where the size of the neighborhood only depends on the bilipshitz constant related to the quasi-geodesic and not on its length. This is due to the negative curvature of hyperbolic spaces and does not hold in Euclidean spaces, for example.

In this paper we investigate the relation between hyperbolicity and geodesic stability for general geodesic metric spaces. For these spaces a concept of hyperbolicity was introduced by M. Gromov [Gro]. See Section 1 below for precise definitions.

It is known that Gromov hyperbolic spaces are geodesically stable. The main result of the present paper shows that the converse is also true. So the notions of Gromov hyperbolicity and geodesic stability are equivalent.

For the proof we use a certain function $G_{X}$ related to a geodesic metric space $X$. According to its geometric interpretation this function may be called the detour growth function. For Gromov hyperbolic spaces it grows at least exponentially. In view of this, it is somewhat surprising that the condition

$$
\lim _{t \rightarrow \infty} \frac{G_{X}(t)}{t}=\infty
$$

\footnotetext{
* Supported as a Feodor Lynen Fellow of the Alexander von Humboldt foundation.
} 
already implies Gromov hyperbolicity as we will see. On the other hand, it can be shown that this condition is equivalent to geodesic stability.

\section{Basic Definitions and Auxiliary Results}

Let $X$ be a metric space. Though the metric on $X$ need not come from a norm, we will denote the distance of two points $x, y \in X$ by $|x-y|$. If $x \in X$ and $M \subseteq X$ then

$$
\operatorname{dist}(x, M)=\inf \{|x-y|: y \in M\} .
$$

The space $X$ is called geodesic, if for two arbitrary points $x, y \in X$ there exists an isometry $\phi:[a, b] \rightarrow X$ of a compact interval $[a, b] \subseteq \mathbf{R}$ into $X$ with $\phi(a)=x$ and $\phi(b)=y$. The image $\operatorname{Im} \phi=\phi([a, b])$ is called a geodesic segment joining $x$ and $y$ and will be denoted by $[x, y]$. Since we do not require uniqueness, this notation is ambiguous, but it is convenient.

A geodesic triangle $\Delta$ is a set $\Delta=\left[x_{1}, x_{2}\right] \cup\left[x_{2}, x_{3}\right] \cup\left[x_{3}, x_{1}\right] \subseteq X$. We say that $\Delta$ satisfies the Rips condition with constant $\delta \geq 0$, if for each permutation $k, l, m$ of the numbers $1,2,3$ and each $x \in\left[x_{k}, x_{l}\right]$ we have $\operatorname{dist}\left(x,\left[x_{k}, x_{m}\right] \cup\left[x_{m}, x_{l}\right]\right) \leq \delta$. In other words, each point on a side of $\Delta$ has distance to the opposite sides less than or equal to $\delta$.

A geodesic metric space $X$ is called $\delta$-hyperbolic for $\delta \geq 0$ if each geodesic triangle satisfies the Rips condition with constant $\delta$. The space $X$ is called Gromov hyperbolic if it is $\delta$-hyperbolic for some $\delta \geq 0$.

Hyperbolicity for general metric spaces was introduced by M. Gromov [Gro]. Our definition is equivalent to Gromov's original definition for geodesic metric spaces (cf. [G-H, ch. 2]).

A path is a continuous mapping $\phi:[a, b] \rightarrow X$ of an interval $[a, b] \subseteq \mathbf{R}$ into the metric space $X$. The length of $\phi$ is defined as

$$
\text { length }(\phi)=\sup _{a=t_{0}<t_{1}<\cdots<t_{n}=b} \sum_{\nu=1}^{n}\left|\phi\left(t_{\nu}\right)-\phi\left(t_{\nu-1}\right)\right| .
$$

Here the supremum is taken over all possible decompositions of the interval $[a, b]$ by points $a=t_{0}<t_{1}<\cdots<t_{n}=b$. As usual we call $\phi$ rectifiable if length $(\phi)<\infty$.

If $[c, d] \subseteq[a, b]$, we denote by $\phi \mid[c, d]$ the restriction of $\phi$ to $[c, d]$. For $\lambda \geq 1$ the path $\phi$ is called a $\lambda$-chord-arc curve if

$$
\text { length }(\phi \mid[c, d]) \leq \lambda|\phi(c)-\phi(d)| \text { for all }[c, d] \subseteq[a, b] .
$$

A $\lambda$-quasi-geodesic segment is the image of a $\lambda$-chord-arc curve.

For $K \geq 1$ a mapping $\phi:[a, b] \rightarrow X$ of an interval $[a, b] \subseteq \mathbf{R}$ is called $K$-bilipshitz, if

$$
\frac{1}{K}|c-d| \leq|\phi(c)-\phi(d)| \leq K|c-d| \quad \text { for } c, d \in[a, b] .
$$


Every $K$-bilipshitz map $\phi$ is a $K^{2}$-chord-arc curve. Conversely, if we reparametrize a $\lambda$-chord-arc curve according to arc length, we get a $\lambda$-bilipshitz map. So as in the introduction we could alternatively define quasi-geodesic segments as images of bilipshitz maps. For our purposes it more convenient to work with chord-arc curves.

For $\varepsilon>0$ the open $\varepsilon$-neighborhood $N_{\varepsilon}(A)$ of a set $A \subseteq X$ is

$$
N_{\varepsilon}(A)=\{x \in X: \operatorname{dist}(x, A)<\varepsilon\} .
$$

The geodesic metric space is called geodesically stable if the following condition is true. For each $\lambda \geq 1$ there exists a constant $M>0$ with the following property. If $\phi:[a, b] \rightarrow X$ is a $\lambda$-chord-arc curve, then there exists a geodesic segment $[\phi(a), \phi(b)]$ with

$$
\operatorname{Im} \phi \subseteq N_{M}([\phi(a), \phi(b)]) .
$$

This means that every $\lambda$-quasi-geodesic segment is contained in a neighborhood of a geodesic segment whose size only depends on the constant $\lambda$ and not on the length of the quasi-geodesic segment.

If $t>0$ and $\phi:[a, b] \rightarrow X$ is a path, we call $\phi$ a $t$-detour if there exists a geodesic segment $[\phi(a), \phi(b)]$ and a point $z \in[\phi(a), \phi(b)]$ with $\operatorname{Im} \phi \cap B(z, t)=\emptyset$. Here $B(z, t)=\{x \in X:|x-z|<t\}$ is the open ball with center $z$ and radius $t$. The detour growth function $G_{X}:(0, \infty) \rightarrow[0, \infty]$ is defined as

$$
G_{X}(t)=\inf \{\text { length }(\phi): \phi \text { is a } t \text {-detour }\} \text { for } t>0 .
$$

We have to allow the value $G_{X}(t)=\infty$ here, since the set of rectifiable $t$-detours may be empty. It is clear that $G_{X}\left(t_{1}\right) \leq G_{X}\left(t_{2}\right)$ for $0<t_{1} \leq t_{2}$. For Gromov hyperbolic spaces the function $G_{X}$ grows at least exponentially as the following proposition shows.

PROPOSITION 1.1 Suppose $X$ is $\delta$-hyperbolic. If $\delta>0$ then $G_{X}(t) \geq 2^{(t-1) / \delta-1}$ 1 for $t>0$. If $\delta=0$, then $G_{X}(t) \equiv \infty$.

This is essentially Lemma 1.6 in [CDP, ch. 3]. For the sake of completeness we give a complete proof.

We need the following lemma (cf. [CDP, ch. 3, Lemma 1.5]).

LEMMA 1.2 Suppose $X$ is $\delta$-hyperbolic and $k \in \mathbf{N}_{0}$. If $n=2^{k}, x_{0}, \ldots, x_{n} \in X$ and $z \in\left[x_{0}, x_{n}\right]$, then $\operatorname{dist}\left(z,\left[x_{0}, x_{1}\right] \cup \cdots \cup\left[x_{n-1}, x_{n}\right]\right) \leq k \delta$.

Proof. The proof is by induction on $k$. The statement is true for $k=0$.

Assume the statement is true for $k=l \in \mathbf{N}_{0}$. Let $n=2^{l+1}$ and suppose we are given points $x_{0}, \ldots, x_{n} \in X$ and $z \in\left[x_{0}, x_{n}\right]$.

Since $X$ is $\delta$-hyperbolic, the geodesic triangle $\left[x_{0}, x_{n}\right] \cup\left[x_{n}, x_{n / 2}\right] \cup\left[x_{n / 2}, x_{0}\right]$ satisfies the Rips condition with constant $\delta$. So there exists a point $z^{\prime} \in\left[x_{0}, x_{n / 2}\right] \cup$ $\left[x_{n / 2}, x_{n}\right]$ with $\left|z-z^{\prime}\right| \leq \delta$. If $z^{\prime} \in\left[x_{0}, x_{n / 2}\right]$ then by induction hypothesis 
$\operatorname{dist}\left(z^{\prime},\left[x_{0}, x_{1}\right] \cup \cdots \cup\left[x_{n / 2-1}, x_{n / 2}\right]\right) \leq l \delta$. Similarly, if $z^{\prime} \in\left[x_{n / 2}, x_{n}\right]$, then $\operatorname{dist}\left(z,\left[x_{n / 2}, x_{n / 2+1}\right] \cup \cdots \cup\left[x_{n-1}, x_{n}\right]\right) \leq l \delta$. This implies $\operatorname{dist}\left(z,\left[x_{0}, x_{1}\right] \cup \cdots \cup\right.$ $\left.\left[x_{n-1}, x_{n}\right]\right) \leq(l+1) \delta$.

PROOF OF PROPOSITION 1.1. Assume first that $\delta>0$. Suppose $t>0$ and let $\phi:[a, b] \rightarrow X$ be a $t$-detour of finite length. There exists a geodesic segment $[\phi(a), \phi(b)]$ and a point $z \in[\phi(a), \phi(b)]$ with $\operatorname{dist}(z, \operatorname{Im} \phi) \geq t$.

Let $k \in \mathbf{N}_{0}$ be the smallest number so that $L=\operatorname{length}(\phi) \leq n=2^{k}$. Then $L \geq 2^{k-1}-1$. There are numbers $a=t_{0}<t_{1}<\cdots<t_{n}=b$ so that length $\left(\phi \mid\left[t_{l-1}, t_{l}\right]\right) \leq 1$ for $l \in\{1, \ldots, n\}$. If $x_{l}=\phi\left(t_{l}\right)$ for $l \in\{0, \ldots, n\}$, then Lemma 1.2 shows that $\operatorname{dist}\left(z,\left[x_{0}, x_{1}\right] \cup \cdots \cup\left[x_{n-1}, x_{n}\right]\right) \leq k \delta$. Therefore,

$$
t \leq \operatorname{dist}(z, \operatorname{Im} \phi) \leq k \delta+1 \text {. }
$$

This implies the first part of the statement.

If $\delta=0$ we use a similar argument, but instead of dividing our curve in pieces of length 1 , we divide in pieces of length $\varepsilon$, where $\varepsilon>0$ is arbitrary.

Using Lemma 1.2 it can be shown that then $\operatorname{dist}(z, \operatorname{Im} \phi) \leq \varepsilon$. It follows that no rectfiable $t$-detour for positive $t$ exists. So $G_{X}(t) \equiv \infty$.

A tripod is a union of three segments $T=\left[0, a_{1}\right] \cup\left[0, a_{2}\right] \cup\left[0, a_{3}\right], a_{1}, a_{2}, a_{3} \in \mathbf{R}^{2}$, which have only the origin in common. The distance of two points $x, y \in T$ is defined to be the length of the shortest path with image contained in $T$ that joins $x$ and $y$.

Every geodesic triangle $\Delta=\left[x_{1}, x_{2}\right] \cup\left[x_{2}, x_{3}\right] \cup\left[x_{3}, x_{1}\right] \subseteq X$ can be mapped onto a tripod so that the restriction of the map to each side of $\Delta$ is an isometry. We will call a map with these properties a tripod map.

To see the existence of a tripod map for each geodesic triangle let

$$
s_{k}=\frac{1}{2}\left(\left|x_{k}-x_{l}\right|+\left|x_{k}-x_{m}\right|-\left|x_{l}-x_{m}\right|\right),
$$

where $k, l, m$ is a permuation of the numbers $1,2,3$. Note that $\left|x_{k}-x_{l}\right|=s_{k}+s_{l}$. For $k \in\{1,2,3\}$ choose segments $\Sigma_{k} \subseteq \mathbf{R}^{2}$ originating from the origin of length $s_{k}$ which have only the origin in common. Now define the map $f$ from $\Delta$ onto the tripod $T=\Sigma_{1} \cup \Sigma_{2} \cup \Sigma_{3}$ as follows. If $x \in \Delta$ there is at least one $k \in\{1,2,3\}$ with $\left|x-x_{k}\right| \leq s_{k}$. Let $f(x) \in T$ be the unique point on the segment $\Sigma_{k}$ with distance $s_{k}-\left|x-x_{k}\right|$ from the origin. The only points for which the choice of $k$ is not unique are mapped onto the origin. So $f$ is well defined and it can easily be seen that $f$ is a tripod map in the above sense.

A tripod map is essentially unique. More precisely, if $f_{1}: \Delta \rightarrow T_{1}$ and $f_{2}$ : $\Delta \rightarrow T_{2}$ are two tripod maps, then there exists an isometry $g: T_{1} \rightarrow T_{2}$ so that $f_{2}=g \circ f_{1}$.

A geodesic triangle $\Delta$ is called $\delta$-thin for $\delta \geq 0$, if there exists a tripod map $f: \Delta \rightarrow T$ with the following property. If $u, v \in \Delta$ and $f(u)=f(v)$, then $|u-v| \leq \delta$. 
LEMMA 1.3 Let $\Delta=\left[x_{1}, x_{2}\right] \cup\left[x_{2}, x_{3}\right] \cup\left[x_{3}, x_{1}\right] \subseteq X$ be a geodesic triangle. If $\Delta$ is $\delta$-thin, then $\Delta$ satisfies the Rips condition with constant $\delta$.

Conversely, if $\Delta$ satisfies the Rips condition with constant $\delta$, then $\Delta i$ is $4 \delta$-thin.

Proof. We adapt the proof in [G-H, ch. 2].

Suppose $\Delta$ is $\delta$-thin. Then there exists a tripod map $f: \Delta \rightarrow T$ so that $u, v \in \Delta$ and $f(u)=f(v)$ imply $|u-v| \leq \delta$. Assume $x$ is a point on one of the sides of $\Delta$. To show that $\Delta$ satisfies the Rips condition with constant $\delta$ we have to find a point $y$ on one of the sides opposite to $x$ with $|x-y| \leq \delta$. Without loss of generality we may assume $x \in\left[x_{1}, x_{2}\right]$ and $\left|x-x_{1}\right| \leq s_{1}$. Here the number $s_{1}$ is defined as in (1.1).

There is a unique point $y \in\left[x_{1}, x_{3}\right]$ with $\left|y-x_{1}\right|=\left|x-x_{1}\right| \leq s_{1}$. Then $f(x)=f(y)$ and so $|x-y| \leq \delta$.

To prove the other part of the lemma note first that for three arbitrary points $a, b, c \in X$ we have

$$
l=\frac{1}{2}(|a-b|+|a-c|-|b-c|) \leq \operatorname{dist}(a,[b, c]) .
$$

To see this let $g: \tilde{\Delta} \rightarrow \tilde{T}$ be a tripod map of the geodesic triangle $\tilde{\Delta}=[a, b] \cup$ $[b, c] \cup[c, a]$ and $w \in[b, c]$ be a point with $\operatorname{dist}(a,[b, c])=|a-w|$. There exists a point $w^{\prime} \in[a, b] \cup[a, c]$ with $g(w)=g\left(w^{\prime}\right)$. Without loss of generality we may assume $w^{\prime} \in[a, b]$. Then

$$
l \leq\left|w^{\prime}-a\right|=|a-b|-|b-w| \leq|a-w|=\operatorname{dist}(a,[b, c]) .
$$

Now assume $\Delta$ satisfies the Rips condition with constant $\delta$, but $\Delta$ is not $4 \delta$-thin. We will show that this leads to a contradiction.

By assumption there exist a tripod map $f: \Delta \rightarrow X$ and points $u, v \in \Delta$ with $f(u)=f(v)$ and $|u-v|>4 \delta$. After a relabeling of the vertices of $\Delta$, if necessary, we may assume that $u \in\left[x_{1}, x_{2}\right], v \in\left[x_{1}, x_{3}\right]$ and $\left|u-x_{1}\right|=\left|v-x_{1}\right| \leq s_{1}$, where $s_{1}$ is the number defined in (1.1). We may even assume $\left|u-x_{1}\right|=\left|v-x_{1}\right|<s_{1}$, since we can shift $u$ and $v$ closer to $x_{1}$.

Then by the above remark

$$
\begin{aligned}
& \operatorname{dist}\left(v,\left[x_{1}, x_{2}\right]\right)= \min \left\{\operatorname{dist}\left(v,\left[x_{1}, u\right]\right), \operatorname{dist}\left(v,\left[u, x_{2}\right]\right)\right\} \\
& \geq \min \left\{\frac{1}{2}\left(\left|v-x_{1}\right|+|v-u|-\left|x_{1}-u\right|\right),\right. \\
& \\
&\left.\quad \frac{1}{2}\left(|v-u|+\left|v-x_{2}\right|-\left|u_{1}-x_{2}\right|\right)\right\} .
\end{aligned}
$$

Now

$$
\begin{aligned}
\left|v-x_{1}\right|+|v-u|-\left|x_{1}-u\right| & =|v-u| \\
|v-u|+\left|v-x_{2}\right|-\left|u-x_{2}\right| & =|v-u|+\left|v-x_{2}\right|-\left(\left|x_{1}-x_{2}\right|-\left|x_{1}-u\right|\right) \\
& =|v-u|+\left(\left|v-x_{2}\right|+\left|x_{1}-v\right|-\left|x_{1}-x_{2}\right|\right) \\
& \geq|v-u| .
\end{aligned}
$$


So $\operatorname{dist}\left(v,\left[x_{1}, x_{2}\right]\right)>2 \delta$. In particular, $\left|v-x_{1}\right|>2 \delta$ and so there exists a point $w \in\left[x_{1}, v\right]$ with $|w-v|=\delta$. Then

$$
\begin{aligned}
\operatorname{dist}\left(w,\left[x_{1}, x_{2}\right]\right) & \geq \operatorname{dist}\left(v,\left[x_{1}, x_{2}\right]\right)-\delta>\delta \\
\operatorname{dist}\left(w,\left[x_{3}, x_{2}\right]\right) & \geq \operatorname{dist}\left(x_{1},\left[x_{2}, x_{3}\right]\right)-\left|x_{1}-w\right| \geq s_{1}-\left|x_{1}-w\right| \\
& >\left|v-x_{1}\right|-\left|w-x_{1}\right|=\delta .
\end{aligned}
$$

Therefore, $\operatorname{dist}\left(w,\left[x_{1}, x_{2}\right] \cup\left[x_{2}, x_{3}\right]\right)>\delta$. But then $\Delta$ cannot satisfy the Rips condition with constant $\delta$.

\section{Statement and Proof of the Main Result}

Now we can state our main result.

THEOREM. Let $X$ be a geodesic metric space. Then the following conditions are equivalent:

(a) $X$ is Gromov hyperbolic,

(b) $X$ is geodesically stable,

(c) $\lim _{t \rightarrow \infty} G_{X}(t) / t=\infty$.

The proof of this theorem is broken up into a series of lemmas and propositions.

LEMMA 2.1 Let $G:(0, \infty) \rightarrow[0, \infty]$ be a function with $\lim _{t \rightarrow \infty} G(t) / t=\infty$. Then there exists a function $f:(0, \infty) \rightarrow(0, \infty)$ with $\lim _{t \rightarrow \infty} f(t) / t=0$ and $\lim _{t \rightarrow \infty} G(f(t)) / t=\infty$.

Proof. Choose a sequence of real numbers $0=x_{0}<x_{1}<\cdots<x_{n}<x_{n+1}<$ . so that $x_{n+1} \geq(n+1) x_{n}$ for $n \in \mathbf{N}_{0}$ and $G(t) \geq n^{2} t$ for $t \geq x_{n}, n \in \mathbf{N}_{0}$. Now define $f(t)=t / n$ for $t \in\left(x_{n}, x_{n+1}\right]$. Then obviously $\lim _{t \rightarrow \infty} f(t) / t=0$. Furthermore, for $t \in\left(x_{n+1}, x_{n+2}\right]$ we have $t /(n+1) \geq x_{n}$ and so

$$
G(f(t))=G\left(\frac{t}{(n+1)}\right) \geq \frac{n^{2}}{n+1} t
$$

This implies $\lim _{t \rightarrow \infty} G(f(t)) / t=\infty$.

LEMMA 2.2 Let $X$ be a geodesic metric space with $\lim _{t \rightarrow \infty} G_{X}(t) / t=\infty$ and suppose $f$ is a function chosen for $G_{X}$ according to Lemma 2.1. If $\Delta \subseteq X$ is a $\delta$-thin geodesic triangle with $\delta$ satisfying $11 \delta<G_{X}(f(\delta))$ and $f(\delta) \leq \delta / 16$, then $\Delta$ is also $8 f(\delta)$-thin.

Proof. Suppose $\Delta=[a, b] \cup[b, c] \cup[c, a] \subseteq X$. Under the assumptions of the lemma we will show that $\Delta$ satisfies the Rips condition with constant $2 f(\delta)$. By Lemma 1.3 this implies that $\Delta$ is $8 f(\delta)$-thin. 
Let $h: \Delta \rightarrow T$ be a tripod map and $p \in[a, b], q \in[b, c], r \in[c, a]$ be the unique points of $\Delta$ which are mapped to the origin by $h$.

We have to show that for each point $x \in \Delta$ there is a point on one of the sides of $\Delta$ opposite to $x$ with distance less to equal to $2 f(\delta)$. Without loss of generality we may assume $x \in[a, p] \subseteq[a, b]$.

We define points $x_{1}, x_{2} \in[a, b]$ as follows. Let $x_{1} \in[a, x] \subseteq[a, b]$ be the unique point with $\left|x_{1}-x\right|=2 \delta$ if $|a-x| \geq 2 \delta$ and let $x_{1}=a$ otherwise. Similarly, let $x_{2} \in[x, b] \subseteq[a, b]$ be the unique point with $\left|x_{2}-x\right|=2 \delta$ if $|b-x| \geq 2 \delta$ and let $x_{1}=b$ otherwise. We consider two cases according to whether $x_{2}$ lies to the 'left' or to the 'right' of $p$.

Case 1. $x_{2} \in[a, p]$. In this case $x_{1}, x, x_{2} \in[a, p]$. Since $h([a, r])=h([a, p])$, there exist points $y_{1}, y_{2} \in[a, r] \subseteq[a, c]$ with $h\left(y_{1}\right)=h\left(x_{1}\right)$ and $h\left(y_{2}\right)=h\left(x_{2}\right)$. Since $\Delta$ is $\delta$-thin, we then have $\left|x_{1}-y_{1}\right| \leq \delta$ and $\left|x_{2}-y_{2}\right| \leq \delta$. Moreover, $\left|y_{1}-y_{2}\right|=\left|x_{1}-x_{2}\right| \leq 4 \delta$.

We want to show that in this situation the assumption $\operatorname{dist}(x,[a, c] \cup[c, b]) \geq$ $f(\delta)$ leads to a contradiction.

Note first that in this case $\left[x_{1}, y_{1}\right] \cap B(x, f(\delta))=\emptyset$.

For if $x_{1}=a$, then $y_{1}=a$ and so

$$
\operatorname{dist}\left(x,\left[x_{1}, y_{1}\right]\right)=|x-a| \geq \operatorname{dist}(x,[a, c] \cup[c, b]) \geq f(\delta) .
$$

If $x_{1} \neq a$, then $\left|x_{1}-x\right|=2 \delta$ and so

$$
\operatorname{dist}\left(x,\left[x_{1}, y_{1}\right]\right) \geq\left|x_{1}-x\right|-\left|x_{1}-y_{1}\right| \geq \delta \geq f(\delta) .
$$

Similarly, $\left[y_{2}, x_{2}\right] \cap B(x, f(\delta))=\emptyset$. Furthermore, if we choose $\left[y_{1}, y_{2}\right] \subseteq[a, c]$,

$$
\operatorname{dist}\left(x,\left[y_{1}, y_{2}\right]\right) \geq \operatorname{dist}(x,[a, c]) \geq f(\delta) .
$$

So we have

$$
\operatorname{dist}\left(x,\left[x_{1}, y_{1}\right] \cup\left[y_{1}, y_{2}\right] \cup\left[y_{2}, x_{2}\right]\right) \geq f(\delta) .
$$

There is a path $\phi$ with endpoints $x_{1}$ and $x_{2}, \operatorname{Im} \phi=\left[x_{1}, y_{1}\right] \cup\left[y_{1}, y_{2}\right] \cup\left[x_{2}, y_{2}\right]$ and

$$
\text { length }(\phi)=\left|x_{1}-y_{1}\right|+\left|y_{1}-y_{2}\right|+\left|y_{2}-x_{2}\right| \leq 6 \delta \text {. }
$$

Since $x \in\left[x_{1}, x_{2}\right] \subseteq[a, b]$, inequality (2.1) shows that $\phi$ is a $f(\delta)$-detour. So by the definition of $G_{X}$ and our assumption on $\delta$ we get

$$
11 \delta<G_{X}(f(\delta)) \leq \text { length }(\phi) \leq 6 \delta .
$$

This is a contradiction.

Case 2. $x_{2} \notin[a, p]$. In this case $x_{1}, x \in[a, p]$ and $x_{2} \in[p, b] \subseteq[a, b]$. Since $h([a, p])=h([a, r])$ and $h([p, b])=h([q, b])$, there exist unique points $y_{1} \in[a, r] \subseteq[a, c]$ and $y_{2} \in[q, b] \subseteq[c, b]$ with $h\left(x_{1}\right)=h\left(y_{1}\right)$ and $h\left(x_{2}\right)=h\left(y_{2}\right)$. 
Since $\Delta$ is $\delta$-thin, we have $\left|y_{1}-x_{1}\right| \leq \delta$ and $\left|y_{2}-x_{2}\right| \leq \delta$. Moreover, $h(q)=h(r)$ and so $|r-q| \leq \delta$. Finally, since $p \in\left[x_{1}, x_{2}\right] \subseteq[a, b]$ and $\left|x_{2}-x_{1}\right| \leq 4 \delta$ we have $\left|y_{1}-r\right|=\left|x_{1}-p\right| \leq 4 \delta$ and $\left|y_{2}-q\right|=\left|x_{2}-p\right| \leq 4 \delta$.

We want to show $\operatorname{dist}(x,[a, c] \cup[c, b]) \leq 2 f(\delta)$. For this we may assume $\operatorname{dist}(x,[a, c] \cup[c, b]) \geq f(\delta)$.

As in Case 1 it can be shown that

$$
\left(\left[x_{1}, y_{1}\right] \cup\left[y_{1}, r\right] \cup\left[q, y_{2}\right] \cup\left[y_{2}, x_{2}\right]\right) \cap B(x, f(\delta))=\emptyset \text {. }
$$

There is a path $\phi$ with endpoints $x_{1}$ and $x_{2}$,

$$
\operatorname{Im} \phi=\left[x_{1}, y_{1}\right] \cup\left[y_{1}, r\right] \cup[r, q] \cup\left[q, y_{2}\right] \cup\left[y_{2}, x_{2}\right]
$$

and

$$
\text { length }(\phi)=\left|x_{1}-y_{1}\right|+\left|y_{1}-r\right|+|r-q|+\left|q-y_{2}\right|+\left|y_{2}-x_{2}\right| \leq 11 \delta .
$$

Since $x \in\left[x_{1}, x_{2}\right]$, the path $\phi$ would be a $f(\delta)$-detour by (2.2), if $[r, q] \cap$ $B(x, f(\delta))=\emptyset$. But then

$$
11 \delta<G_{X}(f(\delta)) \leq \text { length }(\phi) \leq 11 \delta
$$

which is impossible. So there exists a point $z \in[r, q]$ with $|z-x|<f(\delta)$. We want to show that $\operatorname{dist}(z,[a, c] \cup[c, b]) \leq f(\delta)$.

Assume $\operatorname{dist}(z,[a, c] \cup[c, b])>f(\delta)$. Choose $z_{1} \in[r, c] \subseteq[a, c]$ and $z_{2} \in$ $[q, c] \subseteq[b, c]$ with $\left|z_{1}-r\right|=\left|z_{2}-q\right|=3 \delta$ if $|r-c|=|q-c| \geq 3 \delta$ and let $z_{1}=z_{2}=c$ otherwise. In any case $h\left(z_{1}\right)=h\left(z_{2}\right)$ and so $\left|z_{1}-z_{2}\right| \leq \delta$.

There is a continuous path $\psi$ with endpoints $r$ and $q, \operatorname{Im} \psi=\left[r, z_{1}\right] \cup\left[z_{1}, z_{2}\right] \cup$ $\left[z_{2}, q\right]$ and

$$
\text { length }(\psi)=\left|r-z_{1}\right|+\left|z_{1}-z_{2}\right|+\left|z_{2}-q\right| \leq 7 \delta .
$$

If $z_{1}=z_{2}=c$ we have $\operatorname{Im} \psi \subseteq[a, c] \cup[c, b]$ and so

$\operatorname{Im} \psi \cap B(z, f(\delta))=\emptyset$.

If $z_{1}, z_{2} \neq c$, then $\left|z_{1}-r\right|=3 \delta$ and so

$$
\operatorname{dist}\left(z,\left[z_{1}, z_{2}\right]\right) \geq\left|z_{1}-r\right|-|r-z|-\left|z_{2}-z_{1}\right| \geq \delta \geq f(\delta) .
$$

It follows that $\operatorname{Im} \psi \cap B(z, f(\delta))=\emptyset$.

Since $z \in[r, q]$, the path $\psi$ is a $f(\delta)$-detour. But then

$$
11 \delta<G_{X}(f(\delta)) \leq \text { length }(\psi) \leq 7 \delta
$$

which is impossible. Our assumption $\operatorname{dist}(z,[a, c] \cup[c, b])>f(\delta)$ has led to a contradiction and so we must have $\operatorname{dist}(z,[a, c] \cup[c, b]) \leq f(\delta)$. This implies

$$
\operatorname{dist}(x,[a, c] \cup[c, b]) \leq|z-x|+\operatorname{dist}(z,[a, c] \cup[c, b]) \leq 2 f(\delta) .
$$

This shows that $\Delta$ satisfies the Rips condition with constant $2 f(\delta)$ and the proof is complete. 
PROPOSITION 2.3 Suppose $X$ is a geodesic metric space with $\lim _{t \rightarrow \infty} G_{X}(t) / t=$ $\infty$. Then $X$ is Gromov hyperbolic.

Proof. Choose a function $f$ for the function $G_{X}$ according to Lemma 2.1. Since $\lim _{t \rightarrow \infty} f(t) / t=0$ and $\lim _{t \rightarrow \infty} G_{X}(f(t)) / t=\infty$, there exists a number $\rho \geq 0$ with $11 t<G(f(t))$ and $f(t) \leq t / 16$ for $t \geq \rho$.

We claim that every geodesic triangle $\Delta \subseteq X$ is $\rho$-thin.

Since $\Delta$ is a compact subset of $X$ the diameter $\delta_{1}$ of $\Delta$ is finite. Obviously, $\Delta$ is $\delta_{1}$-thin. Define the numbers $\delta_{n}$ for $n \in \mathbf{N}$ recursively by $\delta_{n+1}=8 f\left(\delta_{n}\right)$. Since $\delta_{n+1}=8 f\left(\delta_{n}\right) \leq \delta_{n} / 2$ if $\delta_{n} \geq \rho$, there is a smallest number $k \in \mathbf{N}$ so that $\delta_{k} \leq \rho$.

Starting with $\delta=\delta_{1}$ a repeated application of Lemma 2.2 shows that $\Delta$ is $\delta_{k}$-thin. Since $\delta_{k} \leq \rho$, this proves the claim. Every $\rho$-thin geodesic triangle also satisfies the Rips condition with constant $\rho$ by Lemma 1.3. This implies that the space $X$ is $\rho$-hyperbolic.

LEMMA 2.4 Let $X$ be a geodesic metric space and let $\lambda \geq 1, L \geq 0$ and $\varepsilon>0$ be constants. Assume $\phi_{1}:[a, b] \rightarrow X$ and $\phi_{2}:[c, d] \rightarrow X$ are $\lambda$-chord-arc curves with $\phi_{1}(b)=\phi_{2}(c)$ and length $\left(\phi_{1}\right)+$ length $\left(\phi_{2}\right) \leq L$. Put $\Lambda=L / \varepsilon+2 \lambda+1$.

Then there exists a $\Lambda$-chord-arc curve $\phi$ with endpoints $\phi_{1}(a)$ and $\phi_{2}(d), \operatorname{Im} \phi \subseteq$ $N_{\varepsilon}\left(\operatorname{Im} \phi_{1} \cup \operatorname{Im} \phi_{2}\right)$ and length $(\phi) \leq \operatorname{length}\left(\phi_{1}\right)+\operatorname{length}\left(\phi_{2}\right)$.

This lemma says that two chord-arc curves which have a common endpoint can be joined to a new chord-arc curve with controlled chord-arc constant, if we allow some change of the image domain. The proof depends on a technique of cutting off a possible 'cusp', where the given curves abut.

Proof. Define $a^{\prime}=\inf \left\{s \in[a, b]: \operatorname{dist}\left(\phi_{1}(s), \operatorname{Im} \phi_{2}\right) \leq \varepsilon\right\}$. Note that the set of which the infimum is taken contains $b$, so it is not empty.

Let $x=\phi_{1}\left(a^{\prime}\right)$ and define $d^{\prime}=\sup \left\{s \in[c, d]: \operatorname{dist}\left(x, \phi_{2}(s)\right) \leq \varepsilon\right\}$ and $y=\phi_{2}\left(d^{\prime}\right)$.

Then $|x-y| \leq \varepsilon$. Here $|x-y|=\varepsilon$ unless $a^{\prime}=a$ and $d^{\prime}=d$. We have $\left|\phi_{1}(s)-\phi_{2}(t)\right|>\varepsilon$ for $s \in\left[a, a^{\prime}\right), t \in\left(d^{\prime}, d\right]$.

For a geodesic segment $[x, y]$ joining $x$ and $y$ and for $0 \leq s \leq s_{0}=|x-y|$ let $x(s) \in[x, y]$ be the unique point with $|x(s)-x|=s$. Now we define the path $\phi: I=\left[a, a^{\prime}+s_{0}+d-d^{\prime}\right] \rightarrow X$ as follows

$$
\phi(s)= \begin{cases}\phi_{1}(s) & \text { for } s \in I_{1}=\left[a, a^{\prime}\right), \\ x\left(s-a^{\prime}\right) & \text { for } s \in I_{2}=\left[a^{\prime}, a^{\prime}+s_{0}\right], \\ \phi_{2}\left(s+d^{\prime}-a^{\prime}-s_{0}\right) & \text { for } s \in I_{3}=\left(a^{\prime}+s_{0}, a^{\prime}+s_{0}+d-d^{\prime}\right] .\end{cases}
$$

Then $\operatorname{Im} \phi=\phi_{1}\left(\left[a, a^{\prime}\right]\right) \cup[x, y] \cup \phi_{2}\left(\left[d^{\prime}, d\right]\right)$ and so $\operatorname{Im} \phi \subseteq N_{\varepsilon}\left(\operatorname{Im} \phi_{1} \cup \operatorname{Im} \phi_{2}\right)$. Since $|x-y| \leq$ length $\left(\phi_{1} \mid\left[a^{\prime}, b\right]\right)+$ length $\left(\phi_{2} \mid\left[c, d^{\prime}\right]\right)$, we have

$$
\begin{aligned}
\operatorname{length}(\phi) & =\operatorname{length}\left(\phi_{1} \mid\left[a, a^{\prime}\right]\right)+|x-y|+\operatorname{length}\left(\phi_{2} \mid\left[d^{\prime}, d\right]\right) \\
& \leq \operatorname{length}\left(\phi_{1}\right)+\operatorname{length}\left(\phi_{2}\right) .
\end{aligned}
$$


Finally, to show that $\phi$ is a $\Lambda$-chord-arc curve we take arbitrary numbers $s, t \in I$, $s \leq t$, and consider several cases.

(a) $s, t \in I_{1}$. In this case

$$
\operatorname{length}(\phi \mid[s, t])=\text { length }\left(\phi_{1} \mid[s, t]\right) \leq \lambda\left|\phi_{1}(s)-\phi_{1}(t)\right|=\lambda|\phi(s)-\phi(t)| .
$$

(b) $s \in I_{1}, t \in I_{2}$. Here $\phi(s) \in \phi_{1}\left(\left[a, a^{\prime}\right]\right)$ and $\phi(t) \in[x, y]$. Note that $|x-\phi(t)| \leq|\phi(s)-\phi(t)|$ by the definition of $a^{\prime}$, for otherwise

$$
\begin{aligned}
|\phi(s)-y| & \leq|\phi(s)-\phi(t)|+|\phi(t)-y| \\
& <|x-\phi(t)|+|\phi(t)-y|=|x-y| .
\end{aligned}
$$

It follows that $|\phi(s)-x| \leq|\phi(s)-\phi(t)|+|x-\phi(t)| \leq 2|\phi(s)-\phi(t)|$ and so

$$
\begin{aligned}
\operatorname{length}(\phi \mid[s, t]) & =\operatorname{length}\left(\phi_{1} \mid\left[s, a^{\prime}\right]\right)+|x-\phi(t)| \\
& \leq \lambda|\phi(s)-x|+|\phi(s)-\phi(t)| \\
& \leq(2 \lambda+1)|\phi(s)-\phi(t)| .
\end{aligned}
$$

(c) $s \in I_{1}, t \in I_{3}$. Here $|\phi(s)-\phi(t)| \geq \varepsilon$ which implies

$$
\text { length }(\phi \mid[s, t]) \leq L \leq(L / \varepsilon)|\phi(s)-\phi(t)| \text {. }
$$

(d) $s \in I_{2}, t \in I_{2}$. Here length $(\phi \mid[s, t])=|\phi(s)-\phi(t)|$.

(e) $s \in I_{2}, t \in I_{3}$. This case is analog to case (b).

(f) $s \in I_{3}, t \in I_{3}$. This case is analog to case (a).

The cases exhaust all possibilities and show that $\phi$ is a $\Lambda$-chord-arc curve with the required properties.

LEMMA 2.5 Suppose $X$ is a geodesic metric space, $\phi:[a, b] \rightarrow X$ is a path, $\varepsilon>0$ and $L$ is a constant with $0 \leq$ length $(\phi) \leq L<\infty$. Put $\lambda=8^{1+L / \varepsilon}$. and

Then there exists a $\lambda$-chord-arc curve $\psi$ with endpoints $x=\phi(a)$ and $y=\phi(b)$

$$
\operatorname{Im} \psi \subseteq N_{\varepsilon}(\operatorname{Im} \phi)
$$

Proof. The main idea of the proof is to replace $\phi$ by a 'polygonal' path consisting of geodesic segments and then apply the previous lemma to smooth out the corners where the segments touch.

Let $n \in \mathbf{N}$ be the largest integer with $n \leq 1+L / \varepsilon$. We can find numbers $a=$ $t_{0}<t_{1}<\cdots<t_{n}=b$ so that length $\left(\phi \mid\left[t_{k-1}, t_{k}\right]\right) \leq \varepsilon$ for $k \in\{1, \ldots, n\}$. Define $x_{k}=\phi\left(t_{k}\right)$ for $k \in\{0, \ldots, n\}$ and let $\left[x_{k-1}, x_{k}\right]$ be a geodesic segment joining 
$x_{k-1}$ and $x_{k}$ for $k \in\{1, \ldots, n\}$. We have $\left|x_{k-1}-x_{k}\right| \leq \operatorname{length}\left(\phi \mid\left[t_{k-1}, t_{k}\right]\right) \leq \varepsilon$ and so

$$
\left[x_{0}, x_{1}\right] \cup \cdots \cup\left[x_{n-1}, x_{n}\right] \subseteq \overline{N_{\varepsilon / 2}(\operatorname{Im} \phi)} .
$$

Let $\lambda_{1}=1$ and define $\lambda_{k}$ for $k \in\{2, \ldots, n\}$ recursively by $\lambda_{k+1}=2 n(k+1)+$ $2 \lambda_{k}+1$.

We want to construct $\lambda_{k}$-chord-arc curves $\phi_{k}$ for $k \in\{1, \ldots, n\}$ with the following properties. The curve $\phi_{k}$ has the endpoints $x_{0}$ and $x_{k}$, length $\left(\phi_{k}\right) \leq k \varepsilon$ and

$$
\operatorname{Im} \phi_{k} \subseteq N_{\varepsilon /(2 n)}\left(\operatorname{Im} \phi_{k-1} \cup\left[x_{k-1}, x_{k}\right]\right) .
$$

(Let $\operatorname{Im} \phi_{0}=\emptyset$ ).

To see the existence of these curves take for $\phi_{1}$ an arc length parametrization of the geodesic segment $\left[x_{0}, x_{1}\right]$. Then $\phi_{1}$ has the required properties.

If $\phi_{k}$ for a number $k \in\{1, \ldots, n-1\}$ is constructed as required, apply Lemma 2.4 to the curve $\phi_{k}$ and an arc length parametrization $\gamma_{k}$ of the geodesic segment $\left[x_{k}, x_{k+1}\right]$. Note that $\phi_{k}$ and $\gamma_{k}$ have an endpoint in common, namely $x_{k}$. Since $\lambda_{k} \geq 1$, both are $\lambda_{k}$-chord-arc curves. We have

$$
\text { length }\left(\phi_{k}\right)+\text { length }\left(\gamma_{k}\right) \leq(k+1) \varepsilon
$$

by induction hypothesis. Lemma 2.4 shows the existence of a $\Lambda$-chord-arc curve $\phi_{k+1}$ with endpoints $x_{0}$ and $x_{k+1}$, length $\left(\phi_{k+1}\right) \leq(k+1) \varepsilon$ and

$$
\operatorname{Im} \phi_{k+1} \subseteq N_{\varepsilon /(2 n)}\left(\operatorname{Im} \phi_{k} \cup\left[x_{k}, x_{k+1}\right]\right) .
$$

Here

$$
\Lambda=\frac{(k+1) \varepsilon}{\varepsilon /(2 n)}+2 \lambda_{k}+1=\lambda_{k+1} .
$$

The path $\phi_{k+1}$ has the required properties.

Using (2.4) and (2.5) it follows by induction on $k$ that

$$
\operatorname{Im} \phi_{k} \subseteq N_{(n+k) \varepsilon /(2 n)}(\operatorname{Im} \phi) \quad \text { for } k \in\{1, \ldots, n\} .
$$

Define $\psi=\phi_{n}$. The path $\psi$ has endpoints $x_{0}=x$ and $x_{n}=y$, the inclusion (2.3) is valid as we see from (2.6) for $k=n$ and it is a $\lambda_{n}$-chord-arc curve. From the recursive definition of the numbers $\lambda_{k}$ we infer

$$
\lambda_{k+1} \leq 2 n^{2}+1+2 \lambda_{k} \text { for } k \in\{1, \ldots, n-1\} .
$$

This implies $\lambda_{n} \leq\left(2 n^{2}+2\right) 2^{n} \leq 2^{3 n} \leq 8^{1+L / \varepsilon}$. Therefore, $\psi$ has the required properties.

LEMMA 2.6 Suppose $X$ is a geodesic metric space, $\varepsilon>0, \lambda \geq 1, \phi:[a, b] \rightarrow X$ is a $\lambda$-chord-arc curve and $\psi:[c, d] \rightarrow X$ is a path. Assume $\phi(a)=\psi(c)=x$, $\phi(b)=\psi(d)=y$ and $\operatorname{Im} \psi \subseteq N_{\varepsilon}(\operatorname{Im} \phi)$. Then $\operatorname{Im} \phi \subseteq N_{2 \lambda \varepsilon}(\operatorname{Im} \psi)$. 
Proof. Assume $\operatorname{Im} \phi$ is not contained in $N_{2 \lambda \varepsilon}(\operatorname{Im} \psi)$. Then there exists a number $s \in[a, b]$ so that with $z=\phi(s)$ we have $\operatorname{dist}(z, \operatorname{Im} \psi) \geq 2 \lambda \varepsilon$. Since $x, y \in \operatorname{Im} \psi$ it follows that length $(\phi \mid[a, s]) \geq 2 \lambda \varepsilon$ and length $(\phi \mid[s, b]) \geq 2 \lambda \varepsilon$. Therefore, we can find $r \in[a, s]$ and $t \in[s, b]$ so that length $(\phi \mid[r, s])=\lambda \varepsilon$ and length $(\phi \mid[s, t])=\lambda \varepsilon$.

Consider the three open sets $O_{1}=N_{\varepsilon}(\phi([a, r])), O_{2}=N_{\varepsilon}(\phi([r, t]))$ and $O_{3}=N_{\varepsilon}(\phi([t, b]))$. We have $\operatorname{Im} \psi \subseteq N_{\varepsilon}(\operatorname{Im} \phi)=O_{1} \cup O_{2} \cup O_{3}$. Since

$$
\operatorname{dist}(\phi([r, t]), \operatorname{Im} \psi) \geq \operatorname{dist}(z, \operatorname{Im} \psi)-\lambda \varepsilon \geq \varepsilon,
$$

and so $\operatorname{Im} \psi \cap O_{2}=\emptyset$, this inclusion implies $\operatorname{Im} \psi \subseteq O_{1} \cup O_{3}$.

If $O_{1} \cap O_{3} \neq \emptyset$ there would exist a point $u \in O_{1} \cap O_{3}$. We could then find $a^{\prime} \in[a, r]$ and $b^{\prime} \in[t, b]$ with $\left|\phi\left(a^{\prime}\right)-u\right|<\varepsilon$ and $\left|\phi\left(b^{\prime}\right)-u\right|<\varepsilon$. This implies $\left|\phi\left(a^{\prime}\right)-\phi\left(b^{\prime}\right)\right|<2 \varepsilon$. From the fact that $\phi$ is a $\lambda$-chord-arc curve we now get the contradiction

$$
2 \lambda \varepsilon=\text { length }(\phi \mid[r, t]) \leq \text { length }\left(\phi \mid\left[a^{\prime}, b^{\prime}\right]\right) \leq \lambda\left|\phi\left(a^{\prime}\right)-\phi\left(b^{\prime}\right)\right|<2 \lambda \varepsilon .
$$

So $O_{1}$ and $O_{3}$ are disjoint open sets with $\operatorname{Im} \psi \subseteq O_{1} \cup O_{3}$. Furthermore, $x \in$ $O_{1} \cap \operatorname{Im} \psi$ and $y \in O_{3} \cap \operatorname{Im} \psi$ and so the sets $O_{1} \cap \operatorname{Im} \phi$ and $O_{3} \cap \operatorname{Im} \phi$ are not empty. This is a contradiction, since $\operatorname{Im} \psi$ is a connected set.

COROLLARY 2.7 Suppose $X$ is a geodesic metric space and $\varepsilon>0$ is a constant.

If $\psi:[a, b] \rightarrow X$ a path and $[\psi(a), \psi(b)]$ a geodesic segment with $\operatorname{Im} \psi \subseteq$ $N_{\varepsilon}([\psi(a), \psi(b)])$, then $[\psi(a), \psi(b)] \subseteq N_{2 \varepsilon}(\operatorname{Im} \psi)$.

If $\lambda \geq 1, \phi:[a, b] \rightarrow X$ is a $\lambda$-chord-arc curve and $[\phi(a), \phi(b)]$ is a geodesic segment with $[\phi(a), \phi(b)] \subseteq N_{\varepsilon}(\operatorname{Im} \phi)$, then $\operatorname{Im} \phi \subseteq N_{2 \lambda \varepsilon}([\phi(a), \phi(b)])$.

Proof. A geodesic segment is the image of an interval under an isometry. Isometries of intervals into $X$ are 1-chord-arc curves. The statements of the corollary now follow from Lemma 2.6 .

PROPOSITION 2.8 Suppose $X$ is a geodesic metric space. If $X$ is geodesically stable, then $\lim _{t \rightarrow \infty} G_{X}(t) / t=\infty$.

Proof. Suppose $X$ is geodesically stable, but $G_{X}(t) / t$ does not tend to infinity for $t \rightarrow \infty$. Then there exists a number $0<K<\infty$ and a sequence of numbers $\left(t_{n}\right)_{n \in \mathbf{N}}$ with $\lim _{n \rightarrow \infty} t_{n}=\infty$ so that $G_{X}\left(t_{n}\right)<K t_{n}$ for all $n \in \mathbf{N}$.

By definition of $G_{X}$ there exist a sequence of $t_{n}$-detours $\gamma_{n}:\left[a_{n}, b_{n}\right] \rightarrow X$ with length $\left(\gamma_{n}\right) \leq K t_{n}$. Let $x_{n}=\gamma_{n}\left(a_{n}\right)$ and $y_{n}=\gamma_{n}\left(b_{n}\right)$. For each $n \in \mathbf{N}$ there exists a geodesic segment $\left[x_{n}, y_{n}\right]$ and a point $z_{n} \in\left[x_{n}, y_{n}\right]$ with $\operatorname{Im} \gamma_{n} \cap B\left(z_{n}, t_{n}\right)=\emptyset$.

In particular, $\left|x_{n}-z_{n}\right| \geq t_{n}$ and $\left|y_{n}-z_{n}\right| \geq t_{n}$ and so there exist points $u_{n} \in\left[x_{n}, z_{n}\right] \subseteq\left[x_{n}, y_{n}\right]$ with $\left|u_{n}-z_{n}\right|=t_{n} / 4$ and $v_{n} \in\left[z_{n}, y_{n}\right] \subseteq\left[x_{n}, y_{n}\right]$ with $\left|v_{n}-z_{n}\right|=t_{n} / 4$. For $n \in \mathbf{N}$ there exists a path $\psi_{n}$ with endpoints $u_{n}$ and $v_{n}$, $\operatorname{Im} \psi_{n}=\left[u_{n}, x_{n}\right] \cup \operatorname{Im} \gamma_{n} \cup\left[y_{n}, v_{n}\right]$ and

$$
\text { length }\left(\psi_{n}\right)=\left|u_{n}-x_{n}\right|+\text { length }\left(\gamma_{n}\right)+\left|y_{n}-v_{n}\right| \leq 2 K t_{n} .
$$


Let $\lambda=8^{16 K+1}$. By Lemma 2.5 there exists for each $n \in \mathbf{N}$ a $\lambda$-chord-arc curve $\phi_{n}$ with endpoints $u_{n}$ and $v_{n}$ and $\operatorname{Im} \phi_{n} \subseteq N_{t_{n} / 8}\left(\operatorname{Im} \psi_{n}\right)$. Since $X$ is geodesically stable, there exists a constant $M>0$ so that for every $n \in \mathbf{N}$ there exists a geodesic segment $\left[u_{n}, v_{n}\right]^{\prime}$ with $\operatorname{Im} \phi_{n} \subseteq N_{M}\left(\left[u_{n}, v_{n}\right]^{\prime}\right)$. Here we use the prime to distinguish the geodesic segment $\left[u_{n}, v_{n}\right]^{\prime}$ from the possibly different geodesic segment $\left[u_{n}, v_{n}\right] \subseteq\left[x_{n}, y_{n}\right]$.

By Corollary 2.7 we have $\left[u_{n}, v_{n}\right]^{\prime} \subseteq N_{2 M}\left(\operatorname{Im} \phi_{n}\right)$, and so by the definition of $\phi_{n}$

$$
\left[u_{n}, v_{n}\right]^{\prime} \subseteq N_{2 M+t_{n} / 8}\left(\left[x_{n}, u_{n}\right] \cup \operatorname{Im} \gamma_{n} \cup\left[v_{n}, y_{n}\right]\right) .
$$

Choose $k$ large enough so that $t_{k} / 8>2 M$. We want to show that (2.7) leads to a contradiction for $n=k$.

Since $\left|u_{k}-v_{k}\right|=t_{k} / 2$, there is a unique point $z \in\left[u_{k}, v_{k}\right]^{\prime}$ with $\left|z-u_{k}\right|=$ $\left|z-v_{k}\right|=t_{k} / 4$. From $\operatorname{dist}\left(z_{k}, \operatorname{Im} \gamma_{k}\right) \geq t_{k}$ we get

$$
\operatorname{dist}\left(z, \operatorname{Im} \gamma_{k}\right) \geq t_{k}-\left|z-z_{k}\right| \geq t_{k} / 2 \text {. }
$$

Furthermore,

$$
\operatorname{dist}\left(z,\left[x_{k}, u_{k}\right]\right) \geq t_{k} / 4 \text {. }
$$

For otherwise, there would be a point $w \in\left[x_{k}, u_{k}\right]$ with $|w-z|<t_{k} / 4$. But then

$$
\begin{aligned}
\left|x_{k}-v_{k}\right| & \leq\left|x_{k}-w\right|+|w-z|+\left|z-v_{k}\right|<\left|x_{k}-u_{k}\right|+t_{k} / 4+t_{k} / 4 \\
& =\left|x_{k}-u_{k}\right|+\left|u_{k}-v_{k}\right|=\left|x_{k}-v_{k}\right|,
\end{aligned}
$$

which is a contradiction. Similarly,

$$
\operatorname{dist}\left(z,\left[v_{k}, y_{k}\right]\right) \geq t_{k} / 4 \text {. }
$$

Our choice of $k$ and (2.8), (2.9) and (2.10) show that (2.7) is impossible for $n=k$. This is a contradiction.

PROPOSITION 2.9 Suppose $X$ is a geodesic metric space with $\lim _{t \rightarrow \infty} G_{X}(t) / t=$ $\infty$. Then for every $\lambda \geq 1$ there exists a constant $M$ with the following property. If $\phi:[a, b] \rightarrow X$ is a $\lambda$-chord-arc curve and $[\phi(a), \phi(b)]$ is any geodesic segment joining $\phi(a)$ and $\phi(b)$, then $\operatorname{Im} \phi \subseteq N_{M}([\phi(a), \phi(b)])$.

In particular, $X$ is geodesically stable.

Proof. Assume $f$ is a function chosen for $G_{X}$ according to Lemma 2.1 and let $\lambda \geq 1$ be arbitrary. There exists a number $K>0$ so that $8 \lambda t<G_{X}(f(t))$ and $f(t) \leq t / 2$ for $t \geq K$. We will show that $M=2 \lambda K$ has the required properties.

In view of Corollary 2.7 we have to show that if $\phi:[a, b] \rightarrow X$ is a $\lambda$-chord-arc curve, $x=\phi(a), y=\phi(b)$ and $[x, y]$ is an arbitrary geodesic segment joining $x$ and $y$, then

$$
[x, y] \subseteq N_{K}(\operatorname{Im} \phi) .
$$


To see this put $t_{1}=|x-y|+1$ and define recursively $t_{n+1}=f\left(t_{n}\right)$ for $n \in \mathbf{N}$. Since $f\left(t_{n}\right) \leq t_{n} / 2$ for $t_{n} \geq K$ there is a smallest $k \in \mathbf{N}$ with $t_{k} \leq K$.

We want to show

$$
[x, y] \subseteq N_{t_{n}}(\operatorname{Im} \phi) \text { for } n \in\{1, \ldots, k\}
$$

by induction on $n$.

Since $x \in \operatorname{Im} \phi$ and $[x, y] \subseteq B\left(x, t_{1}\right)$ the inclusion (2.12) is true for $n=1$.

Suppose (2.12) is true for a number $l \in\{1, \ldots, k-1\}$. We want to show that the inclusion is also true for $n=l+1$.

Assume this is not the case.

Then there exists a point $z \in[x, y]$ with $\operatorname{dist}(z, \operatorname{Im} \phi) \geq t_{l+1}$.

Let $u \in[x, z] \subseteq[x, y]$ be the unique point with $|u-z|=2 t_{l}$ if $|x-z| \geq 2 t_{l}$ and let $u=x$ otherwise. Similarly, let $v \in[z, y] \subseteq[x, y]$ be the unique point with $|v-z|=2 t_{l}$ if $|v-y| \geq 2 t_{l}$ and let $v=y$ otherwise.

By induction hypothesis $[x, y] \subseteq N_{t_{l}}(\operatorname{Im} \phi)$ and so there are numbers $c, d \in$ $[a, b]$ with $|\phi(c)-u| \leq t_{l}$ and $|\phi(d)-v| \leq t_{l}$. If $u=x$ or $v=y$ we take $c=a$ or $d=b$, respectively.

We have

$$
\text { length }(\phi \mid[c, d]) \leq \lambda|\phi(c)-\phi(d)| \leq 6 \lambda t_{l} .
$$

Here $[c, d]$ is to be interpreted as $[d, c]$ if $d<c$.

There exists a path $\psi$ with endpoints $u$ and $v, \operatorname{Im} \psi=[u, \phi(c)] \cup \phi([c, d]) \cup$ $[\phi(d), v]$ and

$$
\text { length }(\psi)=|u-\phi(c)|+\text { length }(\phi \mid[c, d])+|\phi(d)-v| \leq 8 \lambda t_{l} .
$$

By our assumption $\phi([c, d]) \cap B\left(z, t_{l+1}\right)=\emptyset$. Furthermore, $[u, \phi(c)] \cap B\left(z, t_{l+1}\right)=$ $\emptyset$. For this follows from our assumption if $x=u=\phi(c)$. If $u \neq x,|u-z|=2 t_{l}$ and so

$$
\operatorname{dist}(z,[u, \phi(c)]) \geq t_{l} \geq f\left(t_{l}\right)=t_{l+1} .
$$

Similarly, $[\phi(d), v] \cap B\left(z, t_{l+1}\right)=\emptyset$.

Thus $\psi$ is a path with endpoints $u, v$ and $\operatorname{Im} \psi \cap B\left(z, t_{l+1}\right)=\emptyset$. Since $z \in[u, v]$, the path $\psi$ is a $t_{l+1}$-detour. The number $k \in \mathbf{N}$ is the smallest number with $t_{k} \leq K$. Since $l \in\{1, \ldots, k-1\}$ we have $t_{l} \geq K$ and so

$$
8 \lambda t_{l}<G_{X}\left(f\left(t_{l}\right)\right)=G_{X}\left(t_{l+1}\right) \leq \operatorname{length}(\psi) \leq 8 \lambda t_{l} .
$$

This is a contradiction.

So the inclusion (2.12) is true for all $n \in\{1, \ldots, k\}$. Since $t_{k} \leq K$, this implies (2.11).

PROOF OF THE THEOREM. Conditions (a) and (c) are equivalent by Proposition 1.1 and Proposition 2.3. By Proposition 2.8 and Proposition 2.9 the conditions 
(b) and (c) are also equivalent.

If $X$ is geodesically stable and $\lambda \geq 1$, then there exists a constant $M>0$ with the following property. If $\phi:[a, b] \rightarrow X$ is a $\lambda$-chord-arc curve and $[\phi(a), \phi(b)]$ is any geodesic segment joining $\phi(a)$ and $\phi(b)$, then $\operatorname{Im} \phi \subseteq N_{M}([\phi(a), \phi(b)])$. This follows from the theorem and Proposition 2.9.

\section{Quasi-geodesics in Gromov Hyperbolic Spaces}

The original motivation for the present investigations was to understand the fact that Gromov hyperbolic spaces are geodesically stable.

Proposition 1.1 and Proposition 2.9 give an independent proof of this fact using the function $G_{X}$. It is not difficult to strengthen the results slightly as to obtain an explicit expression for $M$ in Proposition 2.9 in terms of $\lambda$ and the hyperbolicity constant $\delta$ of the space $X$. In this section we want to present a simple alternate approach.

In agreement with [CDP] we consider a generalization of $\lambda$-chord-arc curves. For $\lambda \geq 1$ and $c \geq 0$ the path $\phi:[a, b] \rightarrow X$ is called a $(\lambda, c)$-chord-arc curve, if

$$
\text { length }(\phi \mid[c, d]) \leq \lambda|\phi(d)-\phi(c)|+c \text { for all }[c, d] \subseteq[a, b] \text {. }
$$

We could have based our definition of geodesic stability on this class of curves. This would not have made much difference in Section 2, but we did not want to obscure the main ideas by more technicalities.

We want to present a simple proof of the following fact.

PROPOSITION 3.1 Let $X$ be a $\delta$-hyperbolic geodesic metric space and let $\phi$ : $[a, b] \rightarrow X$ be $a(\lambda, c)$-chord-arc curve. Then there exists a number $M=$ $M(\delta, \lambda, c)>0$ only depending on $\delta, \lambda$ and $c$ so that $\operatorname{Im} \phi \subseteq N_{M}([\phi(a), \phi(b)])$ for every geodesic segment $[\phi(a), \phi(b)]$.

We can take

$$
\begin{aligned}
M & =M(\delta, \lambda, c) \\
& =(1+8 \delta \lambda)\left(8 \delta \lambda^{2}+12 \delta \lambda+2 \lambda+c\right)+4 \delta \lambda+2 \delta+2 .
\end{aligned}
$$

We need two lemmas for the proof.

LEMMA 3.2 ('Projection Lemma'). Suppose $X$ is a $\delta$-hyperbolic geodesic metric space. Let $x_{1}, x_{2}, y_{1}, y_{2} \in X$ and let $g$ be a geodesic segment. Assume $y_{1}, y_{2} \in g$, $\operatorname{dist}\left(x_{k}, g\right)=\left|x_{k}-y_{k}\right| \geq R$ for $k \in\{1,2\}$ and some $R \geq 0$. Then $\left|x_{1}-x_{2}\right|<$ $2 R-4 \delta$ implies $\left|y_{1}-y_{2}\right| \leq 8 \delta$.

This lemma has the following geometric interpretation. The points $y_{1}, y_{2}$ can be considered as 'projections' of the points $x_{1}, x_{2}$, respectively, onto the geodesic segment $g$. The lemma says that this projection decreases the distance of points 
which have a large distance $R$ from $g$ by a factor proportional to the reciprocal of $R$.

Proof. Choose a point $y_{0} \in g$ with $\left|y_{0}-y_{1}\right|=\left|y_{0}-y_{2}\right|=\frac{1}{2}\left|y_{1}-y_{2}\right|$. We have

$$
\operatorname{dist}\left(y_{0},\left[y_{1}, x_{1}\right] \cup\left[x_{1}, x_{2}\right] \cup\left[x_{2}, y_{2}\right]\right) \leq 2 \delta .
$$

To see this, note that by the definition of $\delta$-hyperbolicity there is a point $v \in$ $\left[y_{1}, x_{2}\right] \cup\left[x_{2}, y_{2}\right]$ with $\left|v-y_{0}\right| \leq \delta$. If $v \in\left[x_{2}, y_{2}\right]$, then (3.2) is true. If $v \in\left[y_{1}, x_{2}\right]$, then the $\delta$-hyperbolicity of $X$ shows that there is a point $v^{\prime} \in\left[y_{1}, x_{1}\right] \cup\left[x_{1}, x_{2}\right]$ with $\left|v-v^{\prime}\right| \leq \delta$. It follows that $\left|y_{0}-v^{\prime}\right| \leq 2 \delta$ and so (3.2) holds in any case.

We cannot have $\operatorname{dist}\left(y_{0},\left[x_{1}, x_{2}\right]\right) \leq 2 \delta$. For then there exists a point $z \in\left[x_{1}, x_{2}\right]$ with $\left|z-y_{0}\right|=\operatorname{dist}\left(y_{0},\left[x_{1}, x_{2}\right]\right) \leq 2 \delta$. Without loss of generality we may assume $\left|z-x_{1}\right| \leq\left|z-x_{2}\right|$. This implies $\left|z-x_{1}\right| \leq \frac{1}{2}\left|x_{1}-x_{2}\right|$. We now conclude

$$
R \leq \operatorname{dist}\left(x_{1}, g\right) \leq\left|x_{1}-z\right|+\left|z-y_{0}\right| \leq \frac{1}{2}\left|x_{1}-x_{2}\right|+2 \delta<R .
$$

This is a contradiction.

So from (3.2) we get dist $\left(y_{0},\left[y_{1}, x_{1}\right] \cup\left[x_{2}, y_{2}\right]\right) \leq 2 \delta$. Without loss of generality we may assume $\operatorname{dist}\left(y_{0},\left[y_{1}, x_{1}\right]\right) \leq 2 \delta$. Then there exists a point $z \in\left[y_{1}, x_{1}\right]$ with $\left|z-y_{0}\right|=\operatorname{dist}\left(y_{0},\left[y_{1}, x_{1}\right]\right) \leq 2 \delta$. Since $\left|x_{1}-y_{1}\right|=\operatorname{dist}\left(x_{1}, g\right) \leq\left|x_{1}-y_{0}\right|$ we have

$$
\left|x_{1}-z\right|+\left|z-y_{1}\right|=\left|x_{1}-y_{1}\right| \leq\left|x_{1}-y_{0}\right| \leq\left|x_{1}-z\right|+\left|z-y_{0}\right|
$$

and so $\left|z-y_{1}\right| \leq\left|z-y_{0}\right| \leq 2 \delta$. Therefore,

$$
\left|y_{1}-y_{2}\right|=2\left|y_{1}-y_{0}\right| \leq 2\left|y_{1}-z\right|+2\left|z-y_{0}\right| \leq 8 \delta \text {. }
$$

LEMMA 3.3 Suppose $X$ is a $\delta$-hyperbolic geodesic metric space. Let $\phi:[a, b] \rightarrow$ $X$ be $a(\lambda, c)$-chord-arc curve and $g$ a geodesic segment. Assume that for $t \in[a, b]$

$$
\operatorname{dist}(\phi(t), g) \geq R=4 \delta \lambda+2 \delta+1 \text {. }
$$

If we put $r_{1}=\operatorname{dist}(\phi(a), g)$ and $r_{2}=\operatorname{dist}(\phi(b), g)$, then

$$
\text { length }(\phi) \leq(1+8 \delta \lambda)\left(\lambda\left(r_{1}+r_{2}\right)+8 \delta \lambda+c\right) \text {. }
$$

Proof. Let $T=1+8 \delta \lambda$ and $L=$ length $(\phi)$. Then there exist a number $n \in \mathbf{N}$ and points $a=t_{0}<t_{1}<\cdots<t_{n}=b \in[a, b]$ with length $\left(\phi \mid\left[t_{k}, t_{k+1}\right]\right)=T$ for $k \in\{0, \ldots, n-2\}$ and length $\left(\phi \mid\left[t_{n-1}, t_{n}\right]\right) \leq T$. We have $n \leq 1+L / T$.

Define $x_{k}=\phi\left(t_{k}\right)$ for $k \in\{0, \ldots, n\}$. Then for $k \in\{0, \ldots, n-1\}$

$$
\begin{aligned}
\left|x_{k+1}-x_{k}\right| & \leq \text { length }\left(\phi \mid\left[t_{k+1}, t_{k}\right]\right) \\
& \leq T=1+8 \delta \lambda=2 R-4 \delta-1<2 R-4 \delta .
\end{aligned}
$$


For $k \in\{0, \ldots, n\}$ choose a point $y_{k} \in g$ with $\operatorname{dist}\left(x_{k}, g\right)=\left|x_{k}-y_{k}\right|$. Then $\left|x_{k}-y_{k}\right| \geq R$ by hypothesis. Lemma 3.2 now shows that $\left|y_{k+1}-y_{k}\right| \leq 8 \delta$ for $k \in\{0, \ldots, n-1\}$. Since $\phi$ is a $(\lambda, c)$-chord-arc curve, we get

$$
\begin{aligned}
L & =\text { length }(\phi) \leq \lambda|\phi(b)-\phi(a)|+c \\
& \leq \lambda\left|x_{n}-y_{n}\right|+\lambda \sum_{k=0}^{n-1}\left|y_{k+1}-y_{k}\right|+\lambda\left|x_{0}-y_{0}\right|+c \\
& \leq \lambda\left(r_{1}+r_{2}+8 \delta n\right)+c \leq \frac{8 \delta \lambda}{T} L+\lambda\left(8 \delta+r_{1}+r_{2}\right)+c .
\end{aligned}
$$

This inequality implies (3.4).

PROOF OF PROPOSITION 3.1. Let $M$ be the number defined in (3.1) and let $g=[\phi(a), \phi(b)]$ be an arbitrary geodesic segment joining $\phi(a)$ and $\phi(b)$. We will show that

$$
\operatorname{dist}(\phi(t), g)<M \text { for } t \in[a, b] .
$$

Let $R$ be the number defined in (3.3). Note that $R \leq M$. Choose $s \in[a, b]$ arbitrarily and put $z=\phi(s)$. If $\operatorname{dist}(z, g)<R$, then (3.5) is true for $t=s$. So we may assume $\operatorname{dist}(z, g) \geq R$.

From the continuity of $\phi$ it follows that there exists a maximal interval $[c, d] \subseteq$ $[a, b]$ with $s \in[c, d]$ and $\operatorname{dist}(\phi(t), g) \geq R$ for $t \in[c, d]$. Then

$$
\operatorname{dist}(\phi(c), g)=R \text { and } \operatorname{dist}(\phi(d), g)=R .
$$

Lemma 3.2 applied to the $(\lambda, c)$-chord-arc curve $\phi \mid[c, d]$ now shows that

$$
\text { length }(\phi \mid[c, d]) \leq(1+8 \delta \lambda)(2 \lambda R+8 \delta \lambda+c) \text {. }
$$

Furthermore,

$$
\operatorname{dist}(\phi(s), g) \leq|\phi(s)-\phi(c)|+\operatorname{dist}(\phi(c), g) \leq \operatorname{length}(\phi \mid[c, d])+R .
$$

Combining this inequality with (3.6) we arrive at (3.5) for $t=s$.

\section{Acknowledgements}

This paper was motivated by a seminar about 'Hyperbolicity à la Gromov'. at the University of Michigan. I would like to express my thanks to William Cherry, Juha Heinonen, Pekka Koskela and Steffen Rohde for interesting discussions. Special thanks go to Fred Gehring for his continuous encouragement and interest in my work, the Department of Mathematics in Ann Arbor for its hospitality and the Alexander von Humboldt foundation for its generous support. 


\section{References}

[CDP] Coornaert, M., Delzant, T. and Papadopoulos, A.: Géométrie et théorie de groups, Les groupes hyperbolique de Gromov, Lecture Notes in Math. 1441, Springer, Berlin, Heidelberg, New York, 1990.

[G-H] Ghys, E. and de la Harpe, P. (eds.): Sur les groupes hyperbolique d' après Mikhael Gromov, Birkhäuser, Boston, 1990.

[Gro] Gromov, M.: Hyperbolic groups, in: Essays in Group Theory, S. M. Gersten (ed.), MSRI Publ. 8, Springer, Berlin, Heidelberg, New York, 1987, pp. 75-263. 